\title{
THE THIRTY-FOURTH SUMMER MEETING OF THE AMERICAN MATHEMATICAL SOCIETY
}

The thirty-fourth summer meeting and twelf th colloquium of the Society were held at Amherst College, Amherst, Massachusetts, from Tuesday to Friday, September 4-7, 1928 , preceded by the summer meeting of the Mathematical Association of America.

The colloquium lectures by Professor A. B. Coble were delivered on Tuesday and Wednesday mornings and Thursday afternoon with seventy-seven members in attendance; a report of these lectures, by Professor H. S. White, will appear in an early issue of this Bulletin. On Thursday morn. ing the Society held sectional sessions for the reading of papers, a Section of Geometry and a Section of Algebra, Analysis, and Foundations. General sessions were held on Thursday afternoon and Friday morning. The joint dinner of the Society and the Association, with Ex-President Olds of Amherst, as toastmaster, was held at the Lord Jeffery Inn on Wednesday evening, with about 150 persons present.

During the latter part of Tuesday afternoon, visiting mathematicians and their friends enjoyed an excursion to Mount Holyoke College where they were entertained at tea; and Wednesday afternoon was devoted to an automobile excursion up the beautiful valley of the Connecticut River. President and Mrs. Pease tendered a reception to the guests on Tuesday evening at the Lord Jeffery Inn. The college dormitories were opened for the visitors, and meals were furnished at the Lord Jeffery Inn near the campus, all at very modest rates. The college and town placed their recreational facilities at the disposal of the mathematicians, a feature which was much appreciated.

Resolutions were passed expressing profound appreciation of the cordial hospitality of the College, and especially of its officers, President Pease, Ex-President Olds, and Dean 
Esty, whose kindness in making the remarkably efficient arrangements for the comfort and convenience of the visitors met with praise on every hand. The beautiful surroundings of the venerable college was another of the features which made this meeting one of the most pleasant in the Society's history.

Greetings were cabled from the Society to the International Mathematical Congress meeting simultaneously at Bologna and a reply was received reciprocating the felicitations.

The attendance included the following one hundred twenty-eight members of the Society:

C. R. Adams, R. B. Adams, Allison, Anderton, Bacon, A. A. Bennett, Theodore Bennett, Blichfeldt, G. A. Bliss, Blumberg, Bradshaw, Brinkmann, H. S. Brown, Bullitt, Cairns, B. H. Camp, C. C. Camp, G. A. Campbell, Carlen, F. E. Carr, Coble, Abraham Cohen, Coolidge, A. H. Copeland, L. P. Copeland, Crathorne, Crawley, Currier, Darkow, H. T. Davis, Decker, Dederick, Dines, Doak, DoBell, Dorwart, Douglas, Dresden, Esty, Feinler, W. B. Ford, Fort, Fry, Gehman, D. C. Gillespie, J. W. Glover, V. G. Grove, M. G. Haseman, E. R. Hedrick, Hickson, Huber, Hurwitz, J. I. Hutchinson, Louis Ingold, Ingraham, Dunham Jackson, Jeffery, M. I. Johnson, R. A. Johnson, Karnow, Kempner, Langer, Langman, Latimer, Leib, Lotka, MacColl, MacDuffee, MacMillan, Maizlish, H. P. Manning, Martin Michie, W. E. Milne, Mode, C. L. E. Moore, T. W. Moore, Moriarty, Richard Morris, F. H. Murray, Neelley, Oakley, Olds, F. W. Perkins, L. R. Perkins, Phalen, Pierpont, C. S. Porter, Ransom, C. J. Rees, R. G. D. Richardson, D. E. Richmond, Rider, H. L. Rietz, Ritt, Robison, E. D. Roe, Schelkunoff, Schoonmaker, Seely, Shaub, Sheffer, Simons, Slobin, Slotnick, A. H. Smith, C. E. Smith, S. E. Smith, W. M. Smith, Sprague, Stetson, Struik, Tamarkin, Eugene Taylor, Thurston, Torrey, Tracey, L. E. Ward, J. H. Weaver, Warren Weaver, M. E. Wells, A. H. Wheeler, R. A. Whelan, H. S. White, Wiener, E. W. Wilson, W. A. Wilson, Yeaton.

The Secretary announced the election of the following forty-four persons to membership in the Society:

Mr. William Vinton Bond, University of Göttingen;

Mr. Moffatt Grier Boyce, Western Reserve University;

Professor Marguerite D. Darkow, Pennsylvania State College;

Professor Prentice Dearing Edwards, Ball Teachers College, Muncie, Indiana;

Mr. Carl M. Erikson, Michigan State Normal College;

Mr. William Welch Flexner, Princeton University;

Professor Robert Jeffrey Hannelly, Phoenix Junior College, Phoenix, Arizona; 
Dr. Harry Gordonson Kaplan, Stuyvesant High School, New York City; Mr. Herman Karnow, University of Colorado;

Mr. Akitsugu Kawaguchi, Hokkaido Imperial University, Sapporo, Japan;

Miss Dorothea Agatha Kern, Swarthmore College;

Miss Edna E. Kramer, Wadleigh High School, New York City;

Dr. Horace C. Levinson, Chicago, Ill.

Assistant Professor Edward James McShane, Wichita, Kansas;

Professor Tatsuo Mori, Naval Academy, Yedajima, Hiroshima-Ken, Japan;

Mr. David Clarence Morrow, University of Chicago;

Mr. Cletus Odia Oakley, University of Illinois;

Professor Kurt Edward Rosinger, Pennsylvania State College;

Mr. Robert Clarence Shook, University of Chicago;

Dr. Francis Raymond Stark, Western Union Telegraph Company, New York City;

Dr. Marie Johanna Weiss, Stockton, California;

Mr. Yue Kei Wong, University of Chicago;

Nominees of the Mathematical Association of America:

Dr. H. W. Bailey, University of Illinois;

Mrs. Mary Hegeler Carus, LaSalle, Illinois;

Dr. C. C. Craig, University of Michigan;

Mr. W. C. Risselman, University of Minnesota;

Professor C. C. Wylie, University of Iowa.

Nominees of the General Electric Company:

Dr. E. J. Berg, Mr. D. H. Harms, Mr. M. L. Henderson, Mr. A. Howard, Mr. W. C. Johnson, Jr., Dr. I. Langmuir, Mr. J. K. Leibing, Mr. R. G. Lorraine, Mr. R. H. Park, Mr. V. Petrovsky, Mr. D. C. Prince, Mr. W. F. Skeats, Mr. J. C. Smith, Mr. J. J. Smith, Mr. I. A. Terry, Mr. L. Wetherill; Nominee of the Prudential Insurance Company:

Mr. Valentine Howell.

Five applications for membership were received.

It was announced that Professor G. H. Hardy, of the University of Oxford, has accepted the invitation of the Society to give the sixth Josiah Willard Gibbs Lecture in connection with the Annual Meeting in New York City, his subject being $A n$ introduction to the theory of numbers.

The following appointments were announced: to represent the Society at the inauguration of President F. B. Robinson, of the College of the City of New York, Professor T. S. Fiske; as Committee on Arrangements for the sixth Gibbs Lecture, Dean H. E. Hawkes and Professor G. W. Mullins; to represent the Society at the International Congress in Bologna, Professors R. C. Archibald, G. D. Birkhoff, H. F. Blichfeldt, 
Edward Kasner, Oswald Veblen, and Virgil Snyder; as Committee on Arrangements for the summer meeting of 1929, Professors A. J. Kempner (chairman), E. L. Dodd, C. A. Hutchinson, R. G. D. Richardson, and C. H. Sisam.

The invitations from Brown University for the summer meeting of 1930 and from the University of Minnesota for the summer meeting of 1931 were accepted.

It was announced that for 1928-29 the National Academy of Sciences had allotted to the Society from funds donated by the General Education Board the sum of $\$ 3500$, to be distributed as follows: Bulletin, $\$ 500$; Transactions, $\$ 1500$; American Journal of Mathematics, $\$ 1500$.

A list of nominations for trustees, and officers and other members of the Council was adopted and ordered printed on the ballot.

The Secretary announced that, in response to the request for copies of No. 6 of volume 33 of the Bulletin, members had volunteered to contribute more than enough to make up the shortage.

To meet the deficit for the current and succeeding years, Professor R. C. Archibald has procured a list of more than one hundred subscribers to a special fund, the amount per year being over thirteen hundred dollars. It was voted to extend to him the hearty thanks of the Council for his timely and untiring efforts in behalf of the finances.

The Council voted to thank Professor Clara E. Smith for her able efforts in the campaign of the Membership Committee.

At a joint meeting of the Trustees of the Mathematical Association and the Council of the Society, there was an informal discussion of various international projects which have been suggested for consideration.

Titles and abstracts of the papers read at this meeting follow below. The papers numbered 1 to 16 were read before the Section of Geometry, Professor Coolidge presiding; those numbered 17-36 before the Section of Algebra, Analysis, and Foundations, Professor Jackson presiding; those numbered 
37 to 45 at the general session on Thursday afternoon, Professor Glover presiding; and those numbered 46 to 70 at the general session on Friday morning, Vice-President Rietz presiding. Professor Errera was introduced by Associate Secretary Dresden, Mr. Feenberg by Professor Ettlinger, and Professor Hufford by Professor Davis. The papers of Altschiller-Court, Ayres, Craig, Dorroh, Edmonson, Ettlinger, Feenberg, Graustein, Gronwall, Hightower, Hollcroft, Kasner, Ketchum, Knebelman, Lane, Lubben, Michal, R. L. Moore, Morse, Putnam, Sharpe, Sheffer (third paper), Simmons, Thomas, Walsh, Whyburn, and N. R. Wilson were read by title.

1. Professor C. L. E. Moore: Linear complexes of curves in Riemannian space.

The complex of curves defined by the equation $\sum \lambda_{i} d x^{i}=0$ has many of the properties of hypersurfaces. In this paper the author investigates some of these similarities, including parallelism, geodesics, normal curvature, mean curvature, geodesic curvature, and torsion. There is a first and a second fundamental form, and consequently Gauss-Codazzi relations, together with the results that depend upon them.

2. Dr. Jesse Douglas (National Research Fellow): Determination of all descriptive and voluminar differential invariants of a space of $K$-spreads.

This paper refers to the author's previous paper, The geometry of systems of $K$-spreads, abstract in this Bulletin, Jan., 1928. The problem is to find all functions of the components of affine connection $\Gamma_{j k}^{i}$ and their partial derivatives which are unchanged (1) under an arbitrary transformation of parameters on the $K$-spreads (descriptive), (2) under a transformation of parameters with constant jacobian (voluminar). It is found that the most general descriptive invariant is a function of a certain fundamental one, $\Pi_{j k}^{i}$, and its derivatives, and the most general voluminar invariant is a function of two fundamental ones, $\Gamma_{a k}^{a}, V_{j k}^{i}$, and their derivatives.

\section{Professor S. A. Schelkunoff: On rotations in ordinary} and null spaces.

The author gives a new proof that the characteristic roots of the ordinary group of rotations are exponential functions of the angles of component simple rotations. He then studies the quasi-orthogonal group. The characteristic roots are still unit complex numbers, but do not, in general, occur in conjugate pairs. Invariant "axes" of quasi-rotations form a quasi-orthogonal set of lines whose direction components are readily determined; the same is true of ordinary rotations as a special case. After the problem of decomposition of general rotations and quasi-rotations 
into simple component rotations is solved, the author passes to the converse problem: to determine the coefficients of a quasi-orthogonal transformation if the direction components of the invariant axes and the corresponding angles of rotations are given. Ordinary rotations are included as a special case. Necessary and sufficient conditions for a set of lines to be a set of invariant axes are then formulated.

4. Professor J. H. Neelley: Concerning covariant forms of the rational plane quartic curve with compound singularities. Second paper.

This paper considers the effects of the tacnode and the oscnode upon covariant forms of the rational plane quartic curve. The curve is treated parametrically, and binary forms which give covariant sets of parameter values are observed. Many cases of multiple roots occur and the corresponding special relations of curve and covariant are discussed. Some curves degenerate and some forms vanish identically. Special attention is given the complete systems of the fundamental pencils of binary quartics, as well as combinants of pencils of line sections for each type of curve.

5. Professor Edward Kasner: Note on the derivative circular congruence of a polygenic function.

This paper appeared in full in the September-October issue of this Bulletin.

\section{Professor W. C. Graustein: On the trisectors of the angles} of a triangle.

A projective method of proof of the theorem in elementary geometry to the effect that the triangle formed by the points of intersection of adjacent trisectors of the internal angles of any triangle is equilateral uncovers 27 equilateral triangles each of which has as vertices points of intersection of trisectors, "internal," "intermediate," or "external," of the given triangle. The 27 equilateral triangles fall into three sets of nine each, and each set of nine into three triads. The vertices of the triangles of a set are, taken collectively, the same for every set, and constitute the 27 finite intersections of three triads of parallel lines. Every two triangles of a triad are perspective, the three centers of perspective of the pairs of triangles of a triad lie on a line, and the three lines of centers of perspective of the three triads of a set go through a point. The three lines are equally spaced about the point in the case of each of two of the three sets, and in the case of the odd set, they are perpendicular to the sides of the given triangle. Finally, the three points of intersection of the lines of centers of perspective for the three sets are collinear.

7. Professor W. C. Graustein: A geometric problem in which order of choice is important.

The answer to the question as to whether, when a collineation between two distinct planes in space is given, it is always possible to move the planes rigidly into new positions in which the lines joining corresponding 
points go through a point, depends on an order of choice. If a metric space is assumed from the start, and a collineation between two finite planes in it is then chosen, the question must be answered in the negative. If the point of departure is a projective space in which there is given a projective correspondence between the points of two planes, it is always possible to introduce the metric (which is necessary to make rigid motions possible) so that the question can be answered affirmatively.

\section{Professor Nathan Altshiller-Court: On five mutually} orthogonal spheres.

The following are some of the results obtained in this paper: (1) If five spheres are mutually orthogonal, each of them is the conjugate sphere of the orthocentric tetrahedron determined by the centers of the remaining four. (2) Conversely: the five conjugate spheres of the five tetrahedra determined by an orthocentric group of five points in space are mutually orthogonal. (3) The sum of the squares of the radii of the five spheres determined by the centers of five given mutually orthogonal spheres is equal to twice the sum of the squares of the radii of the given spheres. (4) The ratio of the square of the radius of one of five mutually orthogonal spheres to the reciprocal of the volume of the tetrahedron determined by the centers of the remaining four is constant in absolute value. (5) The sum of the reciprocals of the squares of the radii of the ten circles along which five such spheres cut is zero. (6) The sum of the squares of the distances of the centers of five such spheres from the center of gravity of these five points is equal to four fifths of the sum of the squares of the radii of the spheres. (7) The tetrahedral pole of a plane of similitude of four of the spheres with respect to the tetrahedron determined by their centers coincides with the pole of the same plane with respect to the fifth sphere.

9. Dr. M. S. Knebelman (National Research Fellow): Groups of motion in related Riemann spaces.

By considering the existence of $r$ linearly independent solutions of Killing's equations, it is shown that if a space admits an $r$-parameter group of motions whose covariant components are $\xi_{i}{ }^{(1)}, \cdots, \xi_{i}{ }^{(r)}$, then every space in geodesic correspondence with it admits an $r$-parameter group of motions whose components are $e^{2 \phi} \xi_{i}(1), \cdots, e^{2 \phi} \xi_{i}(r), \phi$ being the function whose gradient determines the geodesic correspondence. From this theorem that of Beltrami on spaces of constant curvature follows as a simple corollary. By considering the same equations for two conformal spaces, it is shown that if one of them admits an $r$-parameter group of motions, the other admits the same group of motions.

10. Professor F. R. Sharpe: The problem of plane involutions of order $t>2$.

In this paper it is shown that there are three ways of defining a plane involution $I_{t}$ of order $t$ : (1) by the curves of a net of genus $p,(2)$ by the curves of two projective nets, (3) by a pencil of curves of genus $p$, and the algebraically corresponding curves of a linear system of dimension $r$. The 
method (3) gives, for $r>2$, families of involutions which exist for each $p \leqq \frac{1}{2}(t-1)(t-2)$. The method (2) gives single types of $I_{t}$ which exist if, when they are reduced to the form (1), $p \leqq \frac{1}{2} t(t-1)$. The method (1) gives single types with $p \leqq 2 t-2$. A fairly complete study is made for $t=3$ and a less complete discussion is given for $t=4$. The mapping of $I_{t}$ on $S_{r}$, $r \geqq 3$, is also considered. The paper will appear in the American Journal of Mathematics.

11. Professor T. R. Hollcroft: Multiple lines with fixed coincident tangent planes on algebraic surfaces.

Noether derived a formula for the postulation of a multiple line of any given multiplicity on an algebraic surface of given order, but did not treat the cases in which some of the tangent planes to the surface through the line become coincident. The plane section of an algebraic surface containing an $i$-fold line is a curve with an $i$-fold point. An algebraic surface can have as many varieties of $i$-fold lines as an algebraic plane curve has varieties of $i$-fold points. In this paper, there is found the postulation of a line of given multiplicity on an algebraic surface of given order such that the plane section of the surface at the line is any algebraic singularity whose coincident tangents are fixed lines.

12. Professor E. P. Lane: Canonical configurations associated with a surface.

This paper appears in full in the present issue of this Bulletin.

\section{Professor V. G. Grove: Contributions to the general} theory of transformations of nets.

In this paper the author considers further generalizations of the theory of transformations $F, K$, and $\Omega$ of conjugate nets to the general net. In particular he discusses the part that the projective invariant $C$ of Graustein plays in the general theory. The products of transformations $C$ of general nets are considered. The concept of triads of nets is capable of generalization to general nets. Certain of the theorems derived are true generalizations of Slotnick's theorems relative to products of transformations $F$ of conjugate nets. Others are true only for non-conjugate nets.

\section{Dr. M. M. Slotnick: On the projective differential} geometry of conjugate nets.

The analytical work connected with the study of conjugate nets in a projective 3-space can be made symmetrical by introducing a point on the axis congruence associated with the net. The point chosen is the harmonic conjugate of the point of the net with respect to the two focal points of the axis. The complete duality between the axis and ray congruences is analytically evident in a simple way. The results of Wilczynski and Green are obtained very readily. Relations between the point and tangential 
invariants of a net are found by means of which special types of nets are identified in simple analytical manner.

15. Dr. M. M. Slotnick: Semi-parallel maps of lines of curvature.

If the lines of curvature of two surfaces are mapped by a semi-parallel map as defined by Graustein (Annals of Mathematics, (2), vol. 27, pp. 271278), the surfaces are either surfaces of Monge or molding surfaces whose director-developables are either in a Combescurian relation or identical.

16. Professor H. A. Simmons: Affine differential geometry of curves and surfaces. Preliminary communication.

Two results have been obtained in this paper. (i) We have found a complete system of invariants and covariants of plane curves under affine transformations, and have made geometrical interpretations of these invariants and covariants. (ii) We have found a complete system of seminvariants of ruled surfaces under affine transformations. In obtaining result (ii), an incomplete system of partial differential equations was encountered, the completion of which was laborious; in the corresponding projective theory one meets complete systems only. We hope to continue the affine treatment of both curves and surfaces.

17. Professor W. A. Wilson: On irreducible cross-cuts of plane simply, connected regions.

If $R$ is a simply connected plane region whose frontier $K$ is bounded, $H$ is a closed subset of $\bar{R}, m$ and $n$ are points of $R-R \cdot H$, and $H$ separates $m$ from $n$ in $R$ but no closed proper part of $H$ has that property, $H$ is called an irreducible cross-cut of $R$ between $m$ and $n$. If also $H$ does not separate $m$ from $n$ in the plane, it is called regular. If $H$ is an irreducible cross-cut of $R$ between $m$ and $n$, then it is a part of the frontiers of the components of $R-R \cdot H$ containing $m$ and $n$ and $H=\overline{R \cdot H}$; and conversely. The principal results are as follows. If $H$ is a bounded regular irreducible crosscut of $R$ between each pair of $n$ points, $H \cdot K$ is the sum of at least $n$ closed sets and $H$ is a continuum irreducible between each pair of them. If $H \cdot K$ is the sum of $n$ closed sets between each pair of which $H$ is an irreducible continuum, there are at least $n$ components of the complement of $H+K$ whose frontiers contain $H$ as a proper part.

18. Professor R. G. Lubben: Concerning limiting sets in abstract spaces.

The author discusses theorems concerning limiting sets, some of which are extensions of results presented at an earlier meeting of the Society (see this Bulletin, vol. 32 (1926), p. 14). He considers, in particular, necessary and sufficient conditions, both with reference to the collections of point sets and to the spaces in which they are contained, that the limiting sets of such collections should have certain prescribed properties. A property of especial interest is the distributive property: A space is said to have 
the distributive property provided that if in that space $K$ is a closed point set and $G$ is a collection of point sets and each point of $K$ belongs to some subset of $K$ which is the limiting set of some sub-collection of $G$, then $K$ itself is the limiting set of a sub-collection $G_{1}$ of $G$. If it be specified in addition that $G_{1}$ be countable, the space is said to have the countably distributive property. (1) Every regular Hausdorff space having the distributive property and satisfying the first countability axiom is locally compact. (2) In order that a metric space have the countably distributive property it is necessary and sufficient that the space be locally compact and separable.

19. Professor C. G. Latimer: A generalization of Eisenstein's canonical cubic and associated forms.

In this paper we shall consider a certain canonical cubic and associated forms. These forms are defined in exactly the same way as Eisenstein's like-named forms, except that instead of employing a prime $q$, in the form $3 n+1$, we shall use an integer $D$, which contains no square factor, every prime factor of which is in the form $3 n+1$. We shall find that if $a$ is prime to a certain integer, then a necessary and sufficient condition that there exist an associated form belonging to $a$ is that every rational prime factor of $a$ be the norm of a prime ideal in a certain cubic field with the discriminant $D^{2}$. When $D$ is a prime, our results are equivalent to Eisenstein's.

20. Dr. I. M. Sheffer (National Research Fellow): A proof of the fundamental theorem of algebra.

The proof here given, which is believed to be new, is not "elementary," since it makes use of the implicit function theorem and analytic continuation. Yet it may be termed simple, and may possess features of interest.

21. Mr. F. J. Feinler: A reduced Bernoulli polynomial and its properties.

From the initial relation $(B+1)^{n}-B^{n}=0, n \geqq 2$, is derived a set of shortened polynomials. The coefficients show remarkable characteristics, connecting them with Luca's échiquier of $V_{n}$.

22. Dr. W. L. Ayres (National Research Fellow): Concerning the avoidable points of continua.

Urysohn (Mathematische Annalen, vol. 98(1927), p. 301) and Zarankiewicz (Bulletin de l'Académie Polonaise, 1927, p. 194) have defined avoidable points of sets. From each of these strong properties we may obtain a weak property by substituting connected sets for continua. It is shown in this paper that the Urysohn strong and Zarankiewicz weak properties are independent; and both are weaker than the Zarankiewicz strong property and stronger than the Urysohn weak. A point $P$ of a continuum $M$ has the Urysohn weak [strong] property if and only if (1) $M$ is connected im kleinen at $P$, (2) $P$ is not a strong [weak] cut point im kleinen of $M$. 
If every point of a plane continuum $M$ has the Urysohn weak property, then (1) $M$ is a cyclically connected continuous curve such that no two complementary domains of $M$ have a boundary point in common (and conversely), and (2) no countable subset of $M$ cuts $M$. If the point $P$ of a continuum $M$ has the weak Urysohn property with respect to every subcontinuum of $M$, then $P$ has the strong Urysohn property with respect to every subcontinuum of $M$.

\section{Dr. W. L. Ayres: On the end points of continua.}

It is proved that a point $P$ of a continuous curve $M$ is an end point of $M$ if and only if $P$ has the Urysohn weak (or strong) property with respect to every subcontinuum of $M$. An example is given to show that this is not true for either the strong or weak Zarankiewicz properties. This new definition of an end point is examined for its relations in a general continuum to the definitions studied by Gehman (Transactions of this Society, vol. 30(1928), pp. 63-84). It is shown that the set of points of a bounded continuum having Gehman's property 6 is totally disconnected, and an example is given to show that in space of more than two dimensions Gehman's properties 3 and 6 are independent.

24. Dr. W. L. Ayres: Conditions under which every arc of a continuous curve is a subset of a maximal arc of the curve.

Let $M$ denote a bounded plane continuous curve. In order that every arc of $M$ should be a subset of a maximal arc of $M$ either of the following conditions is necessary and sufficient: (1) the set of non-cut points of $M$ of Menger order two should contain no continuum; (2) if $\alpha$ is any arcsegment of $M$ (that is, an arc minus its end points) which is an open subset of $M$, then every point of $\alpha$ should be a cut point of $M$. In order that every arc of $M$ which can be extended in $M$ in both directions should be a subset of an arc of $M$ joining two end points of $M$, either of the following is necessary and sufficient: (1) every point of $M$ which is on the boundary of some complementary domain of $M$ should be a limit point of the cut points of $M$, (2) every point of $M$ which belongs to some simple closed curve that lies in the boundary of some complementary domain of $M$ should be a limit point of the end points of $M$. We shall say that an $\operatorname{arc} \beta$ of $M$ can be extended in both directions in $M$ if there exists an arc $\gamma$ of $M$ such that the $\operatorname{arc} \beta$ is a subset of the arc-segment of $\gamma$.

25. Professor R. G. Putnam: On the separability of classes $(E)$.

R. L. Moore has obtained (Fundamenta Mathematicae, vol. 8, p. 189) theorems concerning the relation between the separability of a class $(D)$ of Fréchet and the proposition that every uncountable point set has a limit point. In the present paper classes $(E)$ are considered, and similar theorems are obtained.

26. Mr. J. L. Dorroh: Concerning a set of axioms for the semi-quadratic geometry of a three-space. 
In his paper Sets of metrical hypotheses for geometry (Transactions of this Society, vol. 9(1908), pp. 487-512), R. L. Moore raised the question whether the semi-quadratic geometry of a three-space is a consequence of the set $O$ of order axioms and the set $C$ of congruence axioms employed therein, together with $M$, the proposition that every segment has a midpoint, and $P_{2}$, a form of the parallel axiom. He stated that this question can be answered in the affirmative if it can be shown that in every space satisfying $O, C$ and $M$, all right angles are congruent to each other. In the present paper it will be shown that all right angles are congruent to each other in every such space. In view of a recent result of the author's, it follows that three-dimensional semi-quadratic geometry follows from $O, C$, and $P_{2}$.

\section{Professor R. L. Moore: On the structure of a continuum.}

For each point $P$ of the bounded plane continuum $M$, let $M_{P_{1}}$ denote the set of all points $X$ of $M$ such that $X$ is not separated from $P$ in $M$ by uncountably many distinct points of $M$, let $M_{P_{2}}$ denote the set of all points $X$ such that $X$ is not separated from $P$ in $M$ by $c$ mutually exclusive point sets each consisting of finitely many points of $M$, let $M_{P_{3}}$ denote the set of all points that can be joined to $P$ by a connected subset of $M$ not containing uncountably many points every two of which are separated in $M$ by a finite point set, and let $M_{P_{4}}$ denote the component containing $P$ of the product of all point sets $g$ such that $g$ contains $P$ and is an element of some upper semi-continuous collection of mutually exclusive continua which fills up $M$ and which is a continuous curve with respect to its elements. For each $i$ let $G_{i}$ denote the collection of all $M_{P_{i}}$ 's for all points $P$ of $M$. Every $G_{i}$ is a continuous curve of elements, $G_{1}$ being dendritic and $G_{3}$ regular. The elements of $G_{4}$ will be called graphatomic subsets of $M$.

\section{Professor G. T. Whyburn: Concerning the cyclic ele- ments of continuous curves.}

The cyclic elements of a continuous curve $M$ of classes (a), (b), and (c) are, respectively, the maximal cyclic curves, cut points, and end points of $M$. In this paper it is shown that for each continuous curve $M$ in $n$-space, a plane continuous curve $K$ exists such that there is a continuous $(1,1)$ correspondence, preserving class, between the cyclic elements of $M$ and the cyclic elements of $K$. There does not exist a general continuous curve $K$ such that, for every continuous curve $M$, a continuous $(1,1)$ correspondence, preserving class, exists between the cyclic elements of $M$ and the cyclic elements of some "subcontinuum of the elements of $K$." Let $M$ be any continuous curve in $n$-space. Then (1) if no two maximal cyclic curves of $M$ have a common point, every two points of $M$ not belonging to the same maximal cyclic curve are separated in $M$ by uncountably many points of $M$; (2) a subset $C$ of $M$ is a connected collection of cyclic elements of $M$ if and only if for each connected subset $H$ of $M, C \cdot H$ is vacuous or connected; and (3) if $C$ is such a collection, and $N$ is any component of $M-C$, then $\bar{C} \cdot \bar{N} \equiv$ one point. 
29. Professor R. U. Hightower: On the classification of the elements of a ring.

A method is presented in this paper for classifying the elements of an abstract ring $R$. The set $C$ of the classes $C_{0}, C_{1}, \cdots$ forms a ring. $C$ contains a subring $\bar{C}$, whose elements, taken together, form a subring $\bar{R}$ of the ring $R$. The class $C_{0}$ is an ideal of $R$ that is in $\bar{R}$. The necessary and sufficient condition that $C_{0}$ be the conductor of $\bar{R}$ relative to $R$ is that $\bar{C}$, which is the ring of classes of $\bar{R}$ modulo $C_{0}$, contain no proper subring $C^{\prime}$ that is invariant in $C$. To each ideal in $R$ prime to $C_{0}$ there corresponds a regular ideal in $\bar{R}$, and conversely. Hence the conditions established by Masazo Sono for unique factorization of ideals in $R$ hold for ideals in $\vec{R}$. Elements are assumed to be abstract. But if $R$, considered as an infinite abelian group with respect to addition, has a finite base, and if $\bar{R}$ is a subgroup of $R$ of finite index, $\bar{R}$ is a ring defined by an algebraic equation.

30. Professor N. R. Wilson: On finding ideals.

This paper is a continuation of a paper in volume 29 of the Transactions of this Society, on finding the basis of an algebraic number field. A similar canonical basis is set up and operations with ideals so expressed are discussed. Congruences for finding the factors of $[p]$, given the field, are then developed, illustrated by the cubic.

31. Professor H. A. Simmons: On a cyclo-symmetric diophantine equation.

In this paper we first show that the cyclo-symmetric equation, (1): $1 /\left(x_{1} x_{2} \cdots x_{r}\right)+1 /\left(x_{2} x_{3} \cdots x_{r+1}\right)+\cdots+1 /\left(x_{n} x_{1} \cdots x_{r-1}\right)=1 / a$, where $a$ is an integer $\geqq 1$, has the solution $\left(x_{1}, x_{2}, \cdots, x_{n}\right) \equiv\left(w_{1}, w_{2}, \cdots, w_{n}\right)$, where $w_{i}=1, \quad(i=1,2, \cdots, \quad r-1), \quad w_{r}=a+1, \quad w_{i}=a w_{1} \cdots w_{i-r}+1$, $(i=r+1, r+2, \cdots, n-1), w_{n}=a w_{1} \cdots w_{n-r}\left(1+w_{n-r+1}+w_{n-r+1} w_{n-r+2}\right.$ $\left.+\cdots+w_{n-r+1} w_{n-r+2} \cdots w_{n-1}\right)$. Next we show if $n>r$, the equation obtained from (1) by merely changing its right member to $b / a$, where $a, b$ are relatively prime positive integers, and $a=(m+1) b-1, m$ being a positive integer, has a solution very much like the one written above. Then we prove special properties of our solutions which cause us to anticipate that they contain the maximum numbers $x$ that can appear in any solutions of the two equations which we consider.

32. Professor L. L. Dines: The resultant of two power series in two variables.

The resultant of two homogeneous polynomials $f(x, y), g(x, y)$ is a certain rational integral function of their coefficients, whose properties are well known. In the present paper the notion of the resultant is extended to two formal power series $f=f_{m}+f_{m+1}+\cdots, g=g_{n}+g_{n+1}+\cdots$, where $f_{i}$ and $g_{i}$ are homogeneous polynomials of degree $i$ in two variables. Many of the properties of the resultant in the algebraic case have analogues in the transcendental case, and these are developed. There are applications in the problem of the analytic implicit functions defined by two equations $F\left(x, y, z_{1}, z_{2}, \cdots, z_{s}\right)=0, G\left(x, y, z_{1}, z_{2}, \cdots, z_{s}\right)=0$. 


\section{Professor L. L. Dines: Linear integral inequalities of} the first kind.

In earlier papers (Transactions of this Society, vol. 30, p. 425, and this Bulletin, vol. 33, p. 695) the author has considered the linear integral inequality $\phi(x)+\int_{a}^{b} k(x, s) \phi(s) d s>0$, and its generalization. The inequality $\int_{a}^{b} k(x, s) \phi(s) d s>0$ presents greater difficulty, as is to be expected in view of the known theory of integral equations of the first kind. It does not possess a relationship to its adjoint integral equation $\int_{a}^{b} \phi(s) k(s, x) d s=0$ similar to that possessed by the inequality previously studied to its adjoint equation. If, however, the inequality symbol $>$ be replaced by the modified symbol >' (meaning "is somewhere greater than and nowhere less than"), the resulting inequality of the first kind admits, at least for restricted classes of kernels, a relationship to its adjoint equation quite similar to that possessed by the inequality previously studied. The theory is extended in the sense of general analysis to the inequality $J k \phi>$ ' 0 .

\section{Professor A. H. Copeland: Admissible numbers in the theory of geometrical probability.}

Geometrical probability is concerned with a set of related events having the power of the continuum. Thus in order to prove the consistency of the fundamental assumptions of geometrical probability, it is necessary to show the existence of a corresponding set of related admissible numbers. Let $\pi(E)$ be the probability that a point, $P$, of an $n$-dimensional continuum, belong to a given set, $E$, where $\pi(E)$ is an absolutely additive function. Then corresponding to every set $E$ it is required to find an admissible number, $x(E)$, belonging to the set $A[\pi(E)]$. The events, $x(E)$, are related as follows. The function $x(E)$ must be absolutely additive and such that $x\left(E_{1}\right) \cdot x\left(E_{2}\right)=0$ whenever $E_{1} \cdot E_{2}=0$. Finally there must exist a fundamental domain, $\Delta$, such that $x(\Delta)=1$. It is proved in this paper that the above restrictions upon the function $x(E)$ cannot all be satisfied for every set $E$, but that these conditions can be satisfied for a very general type of sets $E$. Thus the fundamental assumptions of geometrical probability are consistent provided we apply them to this type of sets $E$.

\section{Professor A. H. Copeland: Independent event histories.}

The condition that a set of numbers (or event histories) be independent is expressed by a certain set of equations which must be satisfied by these numbers. Thus, in a sense, independence is the exception and not the rule. On this account we might expect that, whereas the set of admissible numbers has the power of the continuum, a set of independent numbers must be finite or at most denumerable. However, this is not the case. It is proved in this paper that there exists a set, $E$, of independent admissible numbers such that each of the sets $E \cdot A(p)$, where $0<p<1$, has the power of the continuum, $A(p)$ being the set of admissible events associated with the probability $p$, and $E \cdot A(p)$ being the logical product of the sets $E$ and $A(p)$. From this point of view independence is not exceptional. 
36. Dr. F. W. Perkins: A note on certain continuous nondifferentiable functions.

This note contains a discussion of some properties of a class of functions of which an example of a special type has already been given by the author under the title An elementary example of a continuous non-differentiable function (American Mathematical Monthly, vol. 34(1927), pp. 476 ff.).

37. Professor A. Errera: A theorem about graphs and an application to the theory of numbers.

Certain "incomplete" graphs of the third degree (that is, line graphs whose 0 -cells are incident with one or three 1-cells) can be factored into a graph of first degree (0-cells incident with one 1-cell) and an incomplete graph of the second degree (0-cells incident with one or two 1-cells). The number of factorizations obeys certain laws, and this method can be used to prove a known theorem on Fibonacci numbers $1,3,4,7,11, \cdots$.

38. Professor E. R. Hedrick: Geometric representations of fundamental quantities for non-analytic functions.

In this paper, the author shows how to represent geometrically the values of the usual fundamental quantities $E, F, G$, and the value of the jacobian $J$, for a non-analytic function of a complex variable. These representations lead to geometric proofs of theorems regarding the fundamental quantities, including some whose proofs are ordinarily given otherwise, and some which are new. Thus, the well known theorem regarding the meaning of the equations $E=G, F=0$ appears from the geometric figures.

39. Professor D. J. Struik: Correlation and coherency from the group standpoint.

Correlation theory in statistics is essentially a study of a second degree matrix under certain rotational or affine transformations. These conceptions can be generalized to so-called coherency matrices, of which a very general type has been shown to be useful in quantum mechanics.

40. Dr. T. H. Gronwall: On the wave equation of the hydrogen atom.

This note gives a quite elementary derivation of the non-relativistic set of energy levels and characteristic functions of the hydrogen atom, and it is also proved that the set obtained is complete.

41. Dr. G. A. Campbell: The practical application of the Fourier integral.

The growing practical importance of transients and other non-periodic phenomena makes it desirable to simplify the application of the Fourier integral and to extend the range of problems which the practical man can 
solve in closed form by this method. To this end the known evaluations of Fourier integrals should be brought together in a table. A beginning has been made in the compilation of such a table, the results being presented, however, not as definite integrals but as paired functions, one function being the coefficient for the cisoidal oscillation, $\exp (i 2 \pi f t)$, and the other function the reciprocally related coefficient for the unit impulse. This arrangement simplifies practical applications, since it is the coefficients of which immediate use is made. Many pairs of coefficients of great importance in practical applications exist only as the limits of pairs which are regular in the sense of involving only convergent Fourier integrals. In the compilation a sharp distinction is drawn between these two types of pairs. A natural introduction to pairs seems to be supplied by the parabolic cylinder functions and the Gram-Charlier expansion.

42. Professors H. T. Davis and M. E. Hufford: Note on diffraction and the wave theory of light.

At the April meeting of the Society in Chicago, calculations based on the Lommel theory of diffraction through a circular orifice were presented. A comparison was made between the calculated and experimental values of the radii for a pattern of sixty-six rings. While the mathematical theory predicted the broadening and darkening of the outer rings of the plate, and theory and experiment were in general agreement, individual radii showed as much as 15 per cent error. A re-check of the experiment reveals an error in the wave length used in the first plate, and the agreement between experiment and calculation is now reduced to a negligible per cent. The mathematical interest is in the asymptotic formulas found for the Lommel functions.

43. Professor Marston Morse: Singular points of vector fields under general boundary conditions.

Earlier results of Brouwer, Hopf, Lefschetz, Alexander, Birkhoff, and others, in so far as they dealt with vector fields under boundary conditions assume that the vectors are either all directed exteriorly or all interiorly at the boundary. The author shows how to obtain the index sum of the field under the most general boundary conditions. This sum is evaluated in terms of the Euler characteristic of the given manifold and of the Euler characteristics of certain complexes on the boundary determined by the given field of vectors.

\footnotetext{
44. Professor H. T. Davis: Integral equations of infinite order.

T. Lalesco has studied the problem presented by the solution of the functional equation formed by equating to a known function a linear operator in negative powers of $z$, where $z$ is the differential operator $d / d x$. The present paper shows that the Pincherle-Bourlet generatrix equation used in solving functional equations in positive powers of $z$ also holds for functional equations in negative powers. This fact is used to derive a
} 
general solution of integral equations of infinite order from which certain expansions in the Heaviside calculus of electrical circuit theory come as special cases.

45. Professor H. W. Brinkmann: On quadratic fields in which every genus of ideals contains but one class. Preliminary report.

This preliminary paper deals with the determination of those quadratic imaginary fields which have two genera of ideals, each genus containing one class. Such fields are next in order of simplicity to those in which there is but one class of ideals and in which, therefore, the usual unique factorization theorem holds for integers. Interesting applications to elementary number theory appear as special results of the work.

46. Professors Einar Hille and J. D. Tamarkin: On $a$ general problem of summability of Fourier series. Preliminary communication.

This paper deals with two general classes of regular transformations, the one introduced by Nörlund and the second discussed first by Hurwitz and Silverman and subsequently by Hausdorff. An attempt is made to obtain a classification of these transformations according as the LebesgueHardy theorem (the summability of the Fourier series of an integrable function $f(x)$ to the value $f(x)$ almost everywhere) does or does not hold true.

47. Professor C. C. Camp: Expansions associated with partial differential equations and auxiliary conditions on interior as well as bounding loci. Preliminary communication.

In 1916, C. E. Wilder presented problems in the theory of ordinary differential equations with auxiliary conditions at more than two points. The present paper extends this theory to the case of partial differential equations in $p$ variables with auxiliary conditions which may be made to depend for their solution on $p$ ordinary differential systems each of the Wilder type. In addition the author is extending the treatment to partial differential equations of the type $\sum_{l=1}^{p}\left[\partial u / \partial x_{l}+\lambda a_{l}\left(x_{l}\right) u\right]=0$, where none of the $a_{l}\left(x_{l}\right)$ vanishes. It is believed that the theory is extensible to the case in which the $a_{l}\left(x_{l}\right)$ actually change sign in the intervals considered.

48. Dr. A. J. Lotka: A numerical example in integral equations.

The problem of the distribution-in-time of the progeny of a population element, which has been treated on a former occasion (this Bulletin, 1927, p. 403) by demonstrating the additive property of the Thiele seminvariants for successive generations, can also be solved by means of an integral equation. A complete numerical solution is given. This presents itself as 
an aperiodic component with logarithmic decrement, and a series of superposed damped oscillatory components with incommensurable periods. The constants appearing in the solution are functions of the Thiele seminvariants of the first generation. Problems of the same general character also occur in the computation of the current renewals of manufacturing plant, commercial equipment, etc.

\section{Miss Rose Whelan: Approximate solutions of certain general types of boundary value problems from the standpoint of integral equations. Preliminary communication.}

In this paper, the method of approximating the solutions of a general type of boundary value problem in the theory of linear differential and integro-differential equations by means of associated difference systems is justified. In each case both the boundary value problem and the corresponding difference system are reduced to equivalent Fredholm integral equations and the order of difference of the kernels is found. A treatment is given of the problem of determining the order of the difference between solutions, characteristic numbers, and characteristic solutions of two integral equations when the order of difference between kernels is known.

\section{Professor J. I. Hutchinson: Properties of functions} represented by the Dirichlet series $\sum(a \nu+b)^{-s}$ or by linear combinations of such series.

In this paper, the function, $Z(s)$ defined by the series $\sum(a v+b)^{-s}$, is studied with reference to its zeros and its behavior in certain parts of the complex plane. A formula for the real zeros is given. A critical strip is determined for the imaginary zeros, and some clue as to their distribution is indicated by particular examples. A complete survey is made of the problem of determining linear combinations $f(s)$ of the functions $Z(s)$ for different integral values of $b, a$ being a fixed integer, so as to satisfy functional equations of the type considered by Cahen for $a$ a prime. Various theorems are given concerning the imaginary zeros of the functions $f(s)$, the number of which is proved to be infinite. The functional equations are generalized so as to include the Dirichlet $L$-functions with imaginary characters as special cases.

51. Professor C. R. Adams: On the linear partial qdifference equation of general type.

The equation studied in this paper is (1): $\sum_{i, j=0}^{n} a_{i j}(x, y) f\left(q^{i} x, r^{i} y\right)=0$, in which the $a_{i j}(x, y)$ are known functions of the complex variables $x$ and $y$, analytic at $(0,0) ; q$ and $r$ are constants, not zero and of moduli $\neq 1$; and $f(x, y)$ is to be determined. Series which formally satisfy (1) are found, and direct proof of their convergence is given. In this manner it is shown that in general there exists a one-parameter family of sets of solutions of the equation (1), each set comprising $n$ solutions analytic in the vicinity of $(0,0)$. The situation at $(\infty, \infty)$, or at $(0, \infty)$, or at $(\infty, 0)$, is entirely 
parallel if the coefficient functions $a_{i j}(x, y)$ are analytic there. In addition to the regular case, in which the roots of the characteristic equation are restricted by the usual sort of hypothesis from being multiple, infinite, zero, etc., a large class of irregular cases is treated. The non-homogeneous equation obtained by adding to the left member of (1) a known function $b(x, y)$ analytic at $(0,0)$ is also discussed. The methods employed are applicable to equations like (1) in more than two independent variables.

52. Dr. I. M. Sheffer (National Research Fellow): Expansions in generalized Appell polynomials, and related questions.

By generalized Appell polynomials we mean the polynomials $\left\{G_{n}(x)\right\}$ defined by $e^{t x}\left\{A_{0}(t)+x^{k} A_{1}(t)+\cdots+x^{k} A_{k}(t)\right\}=\sum G_{n}(x) t^{n}$. They make their appearance in the study of certain integral equations in the complex plane. We study expansions in these polynomials of (a suitable class of) analytic functions, and exhibit the relation of these expansions to the solutions of the integral equations mentioned. The theory is intimately tied up with certain linear differential equations of infinite order. The connection between such differential equations and integral equations, which is here brought out, is then utilized to solve two other classes of equations: (1) partial differential equations of infinite order, with constant coefficients; (2) "Laurent" differential equations of infinite order, with constant coefficients.

53. Dr. I. M. Sheffer: Systems, finite and infinite in number, of linear differential equations of infinite order, with constant coefficients.

The author has given existence theorems governing a finite system of linear differential equations of infinite order, with constant coefficients. There the method of attack was one of operators, and by means of suitable differential operators, the system was first reduced to a single equation of infinite order, after which certain solutions of this one equation were shown to represent solutions of the original system. In the present paper we consider the same system of equations, and the extension to systems of infinitely many equations, and show how such systems can be thrown into the form of systems of contour integral equations in the complex plane. These integral equations are then inverted: we obtain solutions which are themselves contour integrals.

\footnotetext{
54. Professor R. L. Jeffery: Sequences of functions which define a definite integral containing a parameter.

This paper is concerned with determining conditions on the bounded function $f(x, y)$ which insure the uniform convergence of the Riemann sum $\sum_{i=1}^{n} f\left(\xi_{i}, y\right) \cdot\left(x_{i}-x_{i-1}\right)$ to its limit $F(y)$, when this limit exists independent of the choice of $\xi_{i}$ on $\left(x_{i-1}, x_{i}\right)$. Sufficient conditions are obtained for the uniformity of this convergence, and these conditions are shown to be
} 
both necessary and sufficient for a certain class of functions. The paper concludes with a consideration of the analogous problem for a RiemannStieltjes sum.

\section{Professor R. L. Jeffery: The existence of the Lebesgue- Stieltjes integral.}

A definition of a Lebesgue-Stieltjes integral of a function $f(x)$ with respect to a non-decreasing function $\phi(x)$ has been given by Hildebrandt (this Bulletin, vol. 24, pp. 188-190) involving the idea of the measurability of a set of points with respect to $\phi(x)$. Making use of a theorem due to Carathéodory (Vorlesungen iiber Reelle Funktionen, \$153) we set $\phi(x)$ $=\nu(x)+\chi(x)$, where $\nu$ depends only on the discontinuities of $\phi$, and where $\chi$ is continuous. It is then shown that the necessary and sufficient condition that every measurable set $E$ be measurable with respect to $\phi$ is that $\chi$ be absolutely continuous. It then follows that if $\chi$ be absolutely continuous, every bounded and measurable function $f(x)$ has a Lebesgue-Stieltjes integral with respect to $\phi$.

56. Professor D. E. Richmond: $A$ new proof of certain relations of Morse in the calculus of variations in the large.

In a recent paper (Transactions of this Society, April, 1928) on the calculus of variations in the large, Morse characterizes the type of an extremal arc whose end points are not conjugate, by the number of mutual conjugate points within the arc. Among other applications, he establishes a set of relations between the numbers $M_{i}$ of extremal arcs of type $i$, which join two given points in an extremal-convex region. The hypotheses include the existence of a field of extremals covering the region of operation. The present paper derives these relations from Morse's hypotheses using only well known continuity properties of the solutions of differential equations. Our methods enable us to state these relations in a somewhat more restrictive form than that given by Morse, and also to gain an insight into the relative position of extremal arcs of different type. It should be stated, however, that Morse has since announced results without the field hypothesis and also for the $n$-dimensional case. Our methods do not appear to apply to these more general cases.

57. Professor H. J. Ettlinger: A countable infinite system of linear differential equations with summable coefficients and the associated difference system.

The system considered in this paper is of the form $y(x)=\int_{0}^{x} A(t) y(t) d t$ $+a$, where $a$ is a vector in a countable infinity of dimensions having length, and $A$ is a matrix with a countable infinity of rows and columns, each element of which is summable (Lebesgue) on $0<x<1$. If $y(x)$ is a vector each of whose components is absolutely continuous on $(0,1)$ and such that the length of $y,|y|$, exists, and $|A y|$ and $|y A|$ are each not greater than $\phi(x)|y|$, where $\phi(x)$ is positive and summable on $(0,1)$, then the given system has a unique solution. The associated difference system is defined 
and solved, and it is shown that the limit of the solution of the difference system is the solution of the system above. The inequality $\left|\int_{0}^{x} y(t) d t\right|$ $\leqq \int_{0}^{x} y|(t)| d t$ is established, and used to show that the method of successive approximations is available in both the algebraic and the differential systems.

58. Mr. E. Feenberg: A countable infinity of ordinary differential equations in abstract space.

A class of scalar functions $H$ of vectors $\omega$, in a countable number of dimensions, including the length function, is defined by the following properties of $H(\omega): H(\omega) \geqq 0, H(0)=0, H\left(\omega_{1}-\omega_{2}\right) \leqq H\left(\omega_{1}\right)+H\left(\omega_{2}\right)$, and $H\left(0,0, \cdots, \omega_{j}, 0,0\right)=H\left(0,0, \cdots, \omega_{i}, \cdots, 0,0\right) \equiv H\left(\omega_{j}\right)$ when $\omega_{i}=\omega_{j}$. In the preceding paper, Professor Ettlinger has given an existence theorem for a linear differential system and an associated difference system in a countable number of variables where the coefficients are summable. The present paper extends the theorem to the following non-linear system: $y(x)=\int_{o}^{x} f(t, y) d t+a$, where $a$ is a constant sector in a countable number of dimensions, $y$ and $f$ are vectors in a countable number of dimensions, and $H(a)$ exists, where $H(\omega)$ is a particular function of the class defined above and on $0 \leqq x_{1} \leqq x_{2} \leqq 1$, we have the following inequalities:

(1) $H\left(\int_{x_{1}}^{x_{2}} f(t, \omega) d t\right) \leqq \int_{x_{1}}^{x_{2}} \phi(t) d t$;

(2) $H\left(\int_{x_{1}}^{x_{2}}\left[f\left(t, \omega_{1}\right)-f\left(t, \omega_{2}\right)\right] d t\right) \leqq \int_{x_{1}}^{x_{2}} \psi(t) H\left(\omega_{1}-\omega_{2}\right) d t$;

(3) $H\left(\int_{x_{1}}^{x_{2}}\left[f_{j}\left(t, \omega_{1}\right)-f_{j}\left(t, \omega_{2}\right)\right] d t\right) \leqq \int_{x_{1}}^{x_{2}} \psi(t) H\left(\omega_{1}-\omega_{2}\right) d t$, where $\phi(x)$ and $\psi(x)$ are positive and summable on $(0,1)$.

59. Mr. H. V. Craig: On the reduction of the Euler equations in the calculus of variations to normal form.

J. $H$. Taylor has given two methods of solving the Euler equations associated with $\int_{t_{1}}^{t_{2}} F(x, \dot{x}) d t$ for the highest derivatives. The present paper modifies these methods so as to apply to the problem containing derivatives of any order.

60. Professor A. D. Michal: The group manifold of finite continuous point and functional transformation groups.

The differential geometry of the manifold of the $r$ parameters of an $r$-parameter continuous group of transformations in $n$ variables $x^{1}, x^{2}, \cdots$, $x^{n}$ has recently been studied by Cartan with the aid of ingenious geometric methods peculiar to his way of looking at an affinely connected manifold. From the analytic and invariant-theoretic standpoint, however, Cartan's geometric methods are by no means the last word on the subject. Furthermore, the proofs of many of his theorems bring into play the variables $x^{1}, \cdots, x^{n}$. In the present paper some of the essential results of Cartan's theory are developed analytically in such a way as to make the theory applicable to finite continuous groups of functional transformations in function space as well as to point transformations in $n$ dimensions. Various fundamental tensor invariants of the group manifold are, for the first time, exhibited in an arbitrary coordinate system. 


\section{Professor J. M. Thomas: Incomplete systems of partial differential equations.}

The theory of a system of total differential equations which is completely integrable is known to correspond exactly to the theory of a jacobian system of linear homogeneous partial differential equations, but the usual treatment of the incompletely integrable case for the two types of systems does not preserve this correspondence. The present paper gives a treatment displaying the equivalence of the two sorts of systems in the incompletely integrable case.

\section{Professor J. M. Thomas: Riquier's existence theorems.}

This paper contains an exposition of the principal results obtained by Riquier in his researches on systems of partial differential equations. It has been offered to the Annals of Mathematics.

63. Professor J. L. Walsh: Note on the expansion of analytic functions in series of polynomials and in series of other analytic functions.

This note continues the study contained in several papers by the author in the Transactions of this Society. The present note gives particular attention to expansions in terms of polynomials associated with an arbitrary Jordan region.

\section{Professor J. L. Walsh: On the degree of approximation} to an analytic function by means of rational functions.

Let $C$ be an arbitrary rectifiable Jordan curve in the z-plane, and let the function $f(z)$ be defined on $C$. A necessary and sufficient condition that $f(z)$ should be regular-analytic on $C$ is that there should exist rational functions $r_{n}(z)$ of degree $2 n, n=0,1, \cdots$, with no poles or limit points of poles on $C$, such that we have $\left|f(z)-r_{n}(z)\right| \leqq M / R^{n}, R>1$, for all $z$ on $C$.

65. Mr. Nat Edmonson: Poisson's integral and plurisegments on the hypersphere.

In a recent paper (American Journal, vol. 49, p. 153) Evans and Bray obtained necessary and sufficient conditions for the existence on the sur face of the unit sphere $S$ of a bounded additive function $F(s)$ of segments, such that $u(M)$, a function harmonic in the interior of $S$, is given by the Stieltjes integral $u(M)=(1 /(4 \pi)) \int S\left\{\left(1-v^{2}\right) .\left(M P^{3}\right)\right\} d F\left(S_{P}\right)$. The present paper extends their results to the hypersphere, in $n$-dimensional space, defined by the parametric e uations $x_{1}=\cos \theta_{1}, x_{2}=\sin \theta_{1} \cos \theta_{2}, \cdots$. $x_{n-1}=\sin \theta_{1} \cdots \sin \theta_{n-2} \cos \theta_{n-1}, \quad x_{n}=\sin \theta_{1} \cdots \sin \theta_{n-2} \sin \theta_{n-1}$. The essential feature is the definition of a system of polar caps and proper segments on $S$ in such a way that an orthogonal system is obtained; the results of Evans and Bray are then readily generalized to $n$ dimensions. The condition (a) of the main theorem of the present paper is eliminated by a method of sequence extraction which is based on the fact that the 
function $F\left(r_{i}, s\right)=\int_{S} u(M) d M$ is of uniformly limited variation; Evans and Bray applied the theory of convergence in the mean to accomplish this end. In other papers now being prepared, the limit approached by $u(M)$ as the point $M$ approaches the boundary of the hypersphere, and functions of configurations analogous to regular curves on the three-dimensional sphere are being studied.

66. Dr. P. W. Ketchum: Analytic functions of hypercomplex variables.

All analytic functions of a hypervariable are monogenic only in case the hypervariable is both associative and commutative. For such hypervariables, all analytic functions are monogenic, and, conversely, all monogenic functions are analytic. Furthermore, the analogues of the two Cauchy integral theorems, Morera's theorem, Taylor's and Laurent's expansions, Mittag-Leffler's theorem, and Weierstrass's factor theorem hold for analytic functions. In this paper, a restricted variable is defined as one whose analytic functions range over a larger class of hypernumbers than the variable itself. The conditions under which all analytic functions of a restricted hypervariable satisfy given differential equations are studied. In particular, variables are obtained all of whose analytic functions satisfy Laplace's equation. The functions of two such harmonic hypervariables are studied in considerable detail. The totality of harmonic functions cannot be obtained from a single hypervariable.

\section{Dr. P. W. Ketchum: A complete solution of Laplace's} equation by an infinite hypervariable.

In this paper, a study is made of the restricted hypervariable $w=x e_{0}$ $+y e_{1}+z e_{-1}$, where $e_{0}, e_{1}$, and $e_{-1}$ are three of an infinite set of units defined by the relations $e_{n}=i^{n} \cos n \alpha, e_{-n}=i^{n} \sin n \alpha$, where $\cos n \alpha$ and $\sin n \alpha$ are to be regarded as hypernumbers and not as a collection of function values. Every analytic function of $w$ is monogenic, and conversely every monogenic function of $w$ is analytic. The line and surface integrals of analytic functions vanish over closed lines and surfaces, respectively, provided they include no singularities. Every analytic function of $w$ satisfies Laplace's equation. With every analytic solution, $f_{0}(x, y, z)$, of Laplace's equation there can be associated an infinite set of functions $f_{k}$ such that $\sum f_{k} e_{k}$ is an analytic function of $w$. The real part of a power series in $w$ is equivalent to an expansion in solid spherical harmonics.

\section{Professor H. A. Simmons: On the ratio of an arc to its chord.}

In this paper it is shown that if $y=f(x)$ represents an arc with a continuously turning tangent, and if $f(x), f^{\prime}(x)$ come under one of the cases (i), (ii), (iii), (iv) below, then $\lim _{c \rightarrow \infty}(s / c)=1, s$ standing for length of arc and $c$ standing for length of corresponding chord: (i) $\lim _{x \rightarrow \infty} f(x)=\infty$, $\lim _{x \rightarrow \infty} f^{\prime}(x)=\infty$; (ii) $\lim _{x \rightarrow a} f(x)=\infty, \lim _{x \rightarrow a} f^{\prime}(x)=\infty$; (iii) $\lim _{x \rightarrow \infty} f(x)=\infty$, $\lim _{x \rightarrow \infty} f^{\prime}(x)=$ const.; (iv) $\lim x_{x \rightarrow \infty} f(x)=$ const., $\lim _{x \rightarrow \infty} f^{\prime}(x)=0$. 


\section{Professor R. E. Langer: On the simultaneous expansion} of arbitrary functions.

This paper is devoted to a discussion of the ordinary differential system $u^{\prime \prime}+p_{1}(x, \rho) u^{\prime}+p_{2}(x, \rho) u=0, \alpha_{i 1}(\rho) u^{\prime}(a)+\alpha_{i 2}(\rho) u(a)+\alpha_{i 3}(\rho) u^{\prime}(b)+\alpha_{i 4}(\rho) u(b)$ $=0, i=1,2$, the coefficients $p_{i}(x, \rho)$ being polynomials of degree $i$ in $\rho$ and the $\alpha_{i j}(\rho)$ also being polynomials. The existing theory for this system asserts the expansibility of an "arbitrary" function in a series of characteristic solutions. It is here shown how two arbitrary functions $f_{1}(x), f_{2}(x)$ may be expanded simultaneously with one determination of coefficients. Of these expansions that for $f_{1}(x)$ corresponds roughly to the expansion previously known, and the discussion of its convergence follows on familiar lines. The associated expansion for $f_{2}(x)$ on the other hand is shown to be convergent to the value $f_{2}(x)$ for an arbitrary choice of the functions $f_{1}(x)$, $f_{2}(x)$, only provided the boundary conditions are peculiarly related to the differential equation. For general boundary conditions of the type above it is shown that the expansion is summable to the value $f_{2}(x)$ by a specified type of Riesz typical means.

70. Professor R. E. Langer: The boundary problem associated with a differential system rational in the parameter.

The asymptotic forms for the solutions of the differential equation $u^{\prime \prime}+r_{1}(x, \rho) u^{\prime}+r_{2}(x, \rho) u=0$ are known provided the coefficients are of the form $r_{i}(x, \rho)=\sum_{j=-i}^{\infty} r_{i j}(x) \rho^{-i}$, and certain other hypotheses are met. The boundary problem associated with this equation and the conditions $\alpha_{i 1} u^{\prime}(a)+\alpha_{i 2} u(a)+\alpha_{i 3} u^{\prime}(b)+\alpha_{i 4} u(b)=0, i=1,2$, the $\alpha_{i j}(\rho)$ being polynomials, has, however, been discussed only for the cases in which the $r_{i}(x, \rho)$ are polynomials of degree $i$ in $\rho$. This paper considers the case in which these cofficients are rational of the form $r_{i}(x, \rho)=\sum_{j-i}^{i} r_{i j}(x) \rho^{-i}$. It is shown that there exists then a double set of characteristic values clustering both at $\rho=\infty$ and at $\rho=0$, and the simultaneous expansions for a double set (four in number) of arbitrary functions with one determination of coefficients is obtained. These expansions are shown to be equiconvergent in pairs with related expansions obtained from associated differential systems which are polynomial in $\rho$.

Arnold DREsDen, Associate Secretary 